\title{
MinI-REVIEW
}

\section{When MAGE meets RING: insights into biological functions of MAGE proteins}

\author{
Yue Feng ${ }^{1,2}$, Jinlan Gao ${ }^{1,3}$, Maojun Yang ${ }^{1,2} \bowtie$ \\ ${ }^{1}$ MOE Key Laboratory of Bioinformatics, Tsinghua University, Beijing 100084, China \\ ${ }^{2}$ Center for Structural Biology, School of Life Sciences, Tsinghua University, Beijing 100084, China \\ ${ }^{3}$ National Laboratory of Medical Molecular Biology, Peking Union Medical College, Tsinghua University, Beijing 100084, China \\ $\triangle$ Correspondence: maojunyang@tsinghua.edu.cn \\ Received December 15, 2010 Accepted December 30, 2010
}

\section{ABSTRACT}

The melanoma antigen (MAGE) family proteins are well known as tumor-specific antigens and comprise more than 60 genes, which share a conserved MAGE homology domain (MHD). Type I MAGEs are highly expressed cancer antigens, and they play an important role in tumorigenesis and cancer cell survival. Recently, several MAGE proteins were identified to interact with RING domain proteins, including a sub-family of E3 ubiquitin ligases. The binding mode between MAGEs and RING proteins was investigated and one important structure of these MAGE-RING complexes was solved: the MAGE-G1NSE1 complex. Structural and biochemical studies indicated that MAGE proteins could adjust the E3 ubiquitin ligase activity of its cognate RING partner both in vitro and in vivo. However, the underlying mechanism was not fully understood. Here, we review these exciting advances in the studies on MAGE family, suggest potential mechanisms by which MAGEs activate the E3 activity of their binding RING proteins and highlight the anticancer potential of this family proteins.

KEYWORDS MAGE, cancer testis antigen, RING, ubiquitin ligase, TRIM28

\section{OVERVIEW OF THE MAGE FAMILY PROTEINS}

The cancer/testis antigens are types of proteins that appear to be only present in germline cells, trophoblasts and tumors (Simpson et al., 2005). It is also implicated that aberrant expression of germline genes in cancer cells might be one of the driving forces of tumorigenesis. The family of cancer/testis antigens has undergone expansion during the past years, and now consists of more than 100 genes, with new members still being identified (Scanlan et al., 2004; Doyle et al., 2010). Cancer/testis antigen genes are widely expressed in various types of tumors, such as melanoma, carcinoma of the bladder and liver (Barker and Salehi, 2002; Miranda, 2010). These immunogenic features have endowed them the potential as therapeutic cancer vaccines (Simpson et al., 2005). Typically, this family is divided into three sub-families: melanoma antigen (MAGE), $G$ antigen (GAGE), and $X$ chromosome antigen (XAGE) families. The majority of these genes exist as multigene families, and are often under similar transcriptional regulation, leading to their co-expression in some contexts (Scanlan et al., 2002).

The first identified member of this family, MZ2-E, was discovered in a patient with melanoma who had cytotoxic $T$ cell recognizing tumor cells (van der Bruggen et al., 1991; Simpson et al., 2005). This gene was later found to belong to a 12-hMAGE-A genes cluster, and thus known as MAGE-A1 (Chomez et al., 2001). Since then, the MAGE family has extensively increased and more than 60 genes have been identified in humans up to now. Based on their different expression patterns, the MAGE family genes are subdivided into two categories. The type I MAGE genes are located in clusters of the $X$ chromosome, and consist of MAGE-A, -B, and $-C$ groups, with their expression restricted to testis, trophoblast, and placenta (Barker and Salehi, 2002; Simpson et al., 2005). Unlike MAGE I genes, type II MAGE genes consist of 15 genes, with undefined chromosome clustering, and are widely expressed in a variety of tissues (Ohman Forslund and Nordqvist, 2001), and not specifically related to cancer. Aberrant expression of MAGE I genes occurs in a wide range of tumors. In addition, type II MAGE genes have also been implicated in many biologic processes, such as neuronal differentiation and apoptosis (Espantman and O'Shea, 2010).

Because of the specific expression in tumors and germline 
cells, but not normal cells, type I MAGE genes have been extensively investigated (Brasseur et al., 1995; De Smet et al., 1995; Patard et al., 1995; Bolli et al., 2002; Brichard and Lejeune, 2007). In normal somatic cells, the promoters of such type I MAGE genes are methylated and thus their expression is suppressed (De Smet et al., 1995). Nevertheless in tumor cells, these genes are aberrantly activated due to the demethylation of their promoters, which is the consequence of a genome-wide demethylation process occurring in many cancers (Miranda, 2010). The abnormal expression of MAGE genes has been observed in a variety of cancer types, including brain, liver, lung, prostate, ovarian, skin, and thyroid (Simpson et al., 2005; Miranda, 2010). Emerging data suggest that type I MAGE antigens modulate cancer cell survival, proliferation, and metastasis processes (Cilensek et al., 2002; Duan et al., 2003; Yang et al., 2007; Liu et al., 2008). For example, recent studies showed that MAGE proteins played an important role in tumor formation in mice. Liu et al. (2008) reported that overexpression of MAGE-A3 promoted cell proliferation and increased primary tumor size, in an orthotopic xenograft model of thyroid cancer. In addition, treatment with MAGE specific small RNAs suppressed melanoma growth in a xenograft mouse model (Yang et al., 2007). The authors suggested that type I MAGE proteins suppress cancer cell apoptosis by suppressing p53 function and thus promote tumorigenesis and tumor progression. Importantly, in vitro acquisition of paclitaxel and doxorubicin resistance in cancer cells could also be associated with increased expression of MAGEs and other cancer/testis antigens (Park et al., 2002; Duan et al., 2003). In a word, type I MAGE proteins display oncogenic properties in promoting cancer cell survival, proliferation, and progression of malignancies. Thus, class I MAGEs became ideal targets for immunotherapies considering their specific expression in a large variety of cancer cells and their contribution to the development of malignancies (Chomez et al., 2001; Simpson et al., 2005). Emerging data have focused on the potential of MAGE proteins as targets of anti-tumor antibodies and as preemptive anticancer vaccines (Brichard and Lejeune, 2007; Goldman and DeFrancesco, 2009).

While the limited expression pattern and potential for immunotherapy of MAGE proteins have been investigated extensively, the more fundamental question concerning the physiologic function and mechanism of MAGE proteins has long been overshadowed. The common feature of the two types of MAGEs is the conservation of a $\sim 170$-amino acid MAGE Homology Domain (MHD) in the center of the molecule (López-Sánchez et al., 2007). The MHD is highly conserved among the MAGE family, with even higher conservation in the subfamilies (Barker and Salehi, 2002; Doyle et al., 2010). For example, the 12 MAGE-A MHDs and the four MAGE-D MHDs have $70 \%$ and $75 \%$ conservation, respectively. Despite the important feature of MAGE proteins, the structure and physiological function of the highly conserved MHD module and how it controls the steps in tumor metastasis have long been elusive. Recently, the structure of this mysterious domain was solved thanks to efforts of structural genomics (PDB: 2WA0). MAGE-A4 MHD was shown to fold in two winged-helix motifs, which are referred to as WH-A and WH-B motif. Based on this, the structure eventually displays its outline for us; however, the physiological function of this conserved domain still remains a mystery.

\section{RING DOMAIN PROTEINS AS BINDING PARTNERS FOR MAGE FAMILY PROTEINS}

It is a powerful approach to define the cellular functions of MAGE family members by identifying the binding partners for the conserved MHDs. Exciting progress in this field was achieved when several groups' data are pointing to RING domain proteins as binding partners for both type I and type II MAGEs (Pebernard et al., 2004; Rual et al., 2005; Sergeant et al., 2005; Yang et al., 2007; Taylor et al., 2008). For example, the interspersed repeat domain of NRAGE/MAGE-D1 (herein referred to as MAGE-D1 for simplicity) was identified to bind RING domain protein XIAP and the avian IAP homolog ITA, members of the inhibitor of apoptosis (IAP) family (Jordan et al., 2001). Through the binding, MAGE-D1 mediates $p 38$ activation and thus facilitates the apoptosis of neural progenitors (Kendall et al., 2005). Two RING domain proteins, Praja1 and Neurodap1, have also been identified to be interacting partners of MAGE-D1 (Sasaki et al., 2002). In addition, the authors also detected the interaction between Praja1 and NECDIN, another member of type II MAGEs. As for type I MAGEs, Yang et al. (2007) suggested that members of all three subfamilies of type I MAGEs form complexes with KAP1/TRIM28 (referred to as TRIM28 hereafter), which is a co-repressor of p53. A genome-wide yeast two-hybrid study has also identified several pairs of binding partners between MAGEs and RING domain proteins (Rual et al., 2005). Consistent with these results, we detected several protein complexes formed by MAGE proteins and RING domain proteins by tandem affinity purification (TAP) coupled to mass spectrometry, and confirmed the results by both coimmunoprecipitation (co-IP) and co-localization studies (Doyle et al., 2010).

RING domain is a cysteine-rich domain that normally forms a cross-brace structure with two zinc ions coordinated in it (Borden, 2000). RING domain proteins are proved to be a big family of E3 ubiquitin ligases, which bind to and localize E2 ubiquitin-conjugating enzymes to substrates and facilitate direct ubiquitin transfer from an E2 to the substrate (Lorick et al., 1999; Jackson et al., 2000). The novel interactions between MAGEs and RING proteins indicated that we could take the protein complex as a whole, rather than looking at MAGEs only as curious antigens expressed on cancer cells.

It is rather interesting to analyze the binding mode between 
the two protein families. In our recent studies, 14 different MAGEs were tested for their ability to bind five different RING domain proteins. As a consequence, MAGEs preferentially bound to one specific RING domain protein and furthermore, similar MAGE proteins appeared to prefer the same RING protein. On the other hand, RING proteins tended to have less binding specificity and one single RING protein bound at least two MAGE proteins (Doyle et al., 2010). The minimal regions required for binding on MAGEs and RINGs were also mapped in three different MAGE-RING complexes, but the outcome was a little surprising. In all three complexes, MAGEs bound RING domain proteins through the conserved MHDs. In contrast, the region on RING domain proteins that bound MAGEs were variable among the three RINGs, but the most conserved RING domain was not required for binding. For example, MAGE-C2 and MAGE-G1 bound the coiled-coil region on TRIM28 and the WH-A motif in NSE1, respectively. Thus, unlike other known protein modules (Pawson and Nash, 2003), the MHD of MAGEs appears to be very complicated and flexible and does not interact with proteins on the common motif, but proteins with the same function. It also indicates that MAGE proteins might function in the ubiquitination cascade mediated by their RING interacting partners (Borden, 2000).

\section{STRUCTURAL INSIGHTS INTO MAGE-RING PROTEIN COMPLEX}

The first and the only structure of these MAGE-RING complexes till now was the MAGE-G1-NSE1 complex solved in our recent study (Doyle et al., 2010). Actually, the nature of this complex is to some extent special, compared with other MAGE-RING protein complexes. Typically, MAGE-G1 and NSE1 are components of the large SMC5/6 complex, which is involved in genomic stability and promotes homologous recombination-mediated DNA repair (Nasmyth and Haering, 2005; Pebernard et al., 2008; Potts, 2009). The human SMC5/6 complex comprises six components: SMC5, SMC6, and NSE (№n-SMC Elements) 1-4. MAGE-G1 was identified to be the human ortholog of NSE3 (Taylor et al., 2008; Potts, 2009). Thus, the MAGE-G1-NSE1 complex is special in that they actually represent a sub-complex within the larger SMC5/6 complex.

Within the structure, the MAGE-G1 MHD (78-295 amino acids) formed two winged-helix motifs (WH-A and WH-B) just as in the structure of MAGE-A4 (PDB: 3NW0). However, a striking difference resides in the relative orientation of the $\mathrm{WH}$ motifs in the NSE1-bound MAGE-G1 and the free MAGE-A4, indicating that MAGE-G1 MHD might undergo a conformational change upon binding NSE1. Interestingly, NSE1 also folded in two WH motifs (referred to as WH-A and WH-B) and a conserved carboxyl-terminal RING domain. Consistent with the mapping experiments, the interaction requires the $\mathrm{WH}-\mathrm{A}$ motif of both proteins, but not the RING domain of NSE1. In the structure, they interact through a large hydrophobic binding interface, with more than $1000 \AA$ from both MAGEG1 and NSE1 buried at the binding interface. A di-leucine motif on MAGE-G1 (L96 and L97) contributes to NSE1 interaction and constitutes partially the hydrophobic core in the MAGE-G1 WH-A motif. Furthermore, L97 and L96 of MAGE-G1 are conserved within the MAGE family (Doyle et al., 2010). Mutation of this motif on MAGE-G1 to alanines disrupted the binding between the two proteins, indicating their important roles in the interaction interface. In addition, mutations of these conserved residues in MAGEs to alanines completely blocked MAGE-B18-LNX1 and MAGE-C2TRIM28 binding both in vitro and in vivo (Doyle et al., 2010). Though there are different motifs to bind the MAGE proteins from the RINGs, the MAGEs most likely use the same binding motif to bind them. Taken together, this structure could represent a common mode of MAGE-RING protein complex and shed light on the functional studies into the conserved MHD in the MAGEs.

\section{FUNCTION AND MECHANISM OF MAGE-RING COMPLEXES}

RING domain is associated with ubiquitin ligase activity in most proteins (Zheng et al., 2002). Thus, it is interesting to examine whether MAGE family proteins function in the E3 ligase activity of their cognate RING domain proteins. Recent evidence showed that MAGE-G1 and MAGE-C2 could enhance the E3 ubiquitin ligase activity of NSE1 and TRIM28, respectively (Doyle et al., 2010). NSE1 displayed rather weak ubiquitin ligase activity on its own (Pebernard et al., 2008). However, the activity of NSE1 could be heavily enhanced by the addition of MAGE-G1, detected by NSE1's auto-ubiquitination. Furthermore, this enhancement relied on the interaction between the two proteins, since the MAGE-G1 L96A/L97A mutant lost the enhancement effect. In the case of MAGE-C2-TRIM28 complex, MAGE-C2 could not only induce the ubiquitin ligase activity of TRIM28 itself, but also enhance the ubiquitination of its substrate p53 by TRIM28 (Wang et al., 2005; Doyle et al., 2010). In addition, Yang et al. (2007) also showed that type I MAGEs binding to TRIM28 suppressed p53-dependent apoptosis in cancer cells. Similarly, mutations of the two conserved leucines on MAGE-C2 also blocked the enhancement effect, indicating a common mode of MAGE proteins binding and activity enhancing of E3 RING ligases.

An important question is how it is achieved that the E3 ubiquitin ligase activities of RING domain proteins are activated by MAGEs, especially in the case of MAGE-C2TRIM28 complex. A strong hint is that MAGE-A2 and MAGE$\mathrm{C} 2$ directly bind to $\mathrm{UbcH} 2$, the same $\mathrm{E} 2$ ubiquitin-conjugating enzyme in the ubiquitination cascade mediated by TRIM28 (Doyle et al., 2010). Thus, a proposed mechanism is that MAGE proteins specifically bind to E2 conjugating enzyme, in which way they help recruit E2 to the E3-substrate complex. 

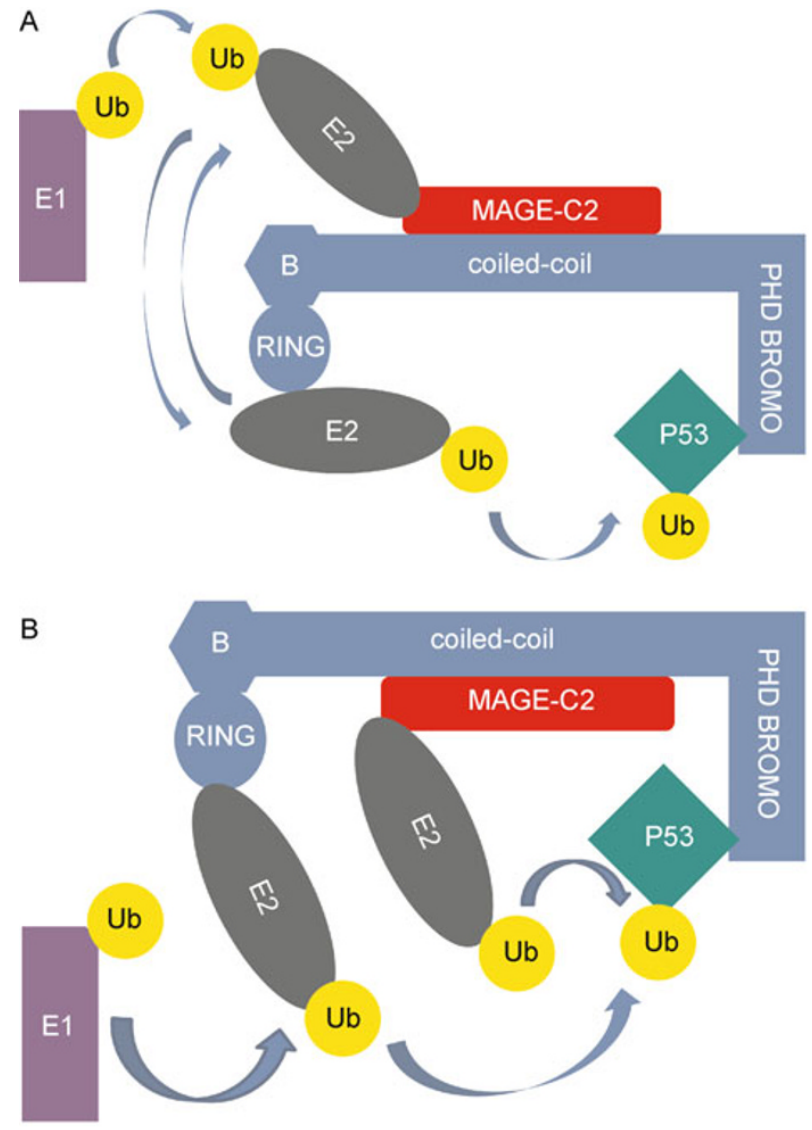

Figure 1. Two proposed models for the mechanism by which the E3 ligase activity of TRIM28 is activated through MAGE-C2 binding. (A) In the first model, the binding of TRIM28 and $\mathrm{UbcH} 2$ (an E2 enzyme) with MAGE-C2 are mutually exclusive. After transferring one ubiquitin to the substrate, an E2 molecule could be recharged by E1 ubiquitin-activating enzyme and at the same time in the vicinity of the whole E3 ligase machinery because of its interaction with MAGE-C2. That is, MAGE-C2 might facilitate the on-site recharging of the E2 conjugating enzyme. (B) In the second model, MAGE-C2 may bind to TRIM28 and $\mathrm{UbcH} 2$ at the same time. Thus, two E2 molecules will be recruited to the TRIM28 machinery, one by the RING domain of TRIM28, and the other by MAGE-C2. In this case, MAGE-C2 might promote the sequential assembly of a polyubiquitin chain on the substrate, with two E2 enzymes transferring ubiquitin molecules onto the substrate one by one.

Importantly, it remains to be answered whether MAGE-C2 could bind to $\mathrm{UbcH} 2$ and TRIM28 at the same time or the two interactions are mutually exclusive. Based on the answer to this question, two models could be proposed. In the first model, after transferring one ubiquitin to the substrate, an E2 molecule could be recharged by E1 ubiquitin-activating enzyme but at the same time in the vicinity of the E3 machinery because of its interaction with MAGE-C2 (Fig. 1A). In this model, MAGE-C2 might facilitate the on-site recharging of the E2 conjugating enzyme. In the second model, MAGE$\mathrm{C} 2$ may bind to TRIM28 and $\mathrm{UbcH} 2$ at the same time. Thus, two E2 molecules will be recruited to the TRIM28 machinery, with one by the RING domain of TRIM28, and the other by MAGE-C2 (Fig. 1B). In this case, MAGE-C2 might promote the sequential assembly of a polyubiquitin chain on the substrate, with two E2 enzymes transferring ubiquitin molecules onto the substrate one by one. However, the exact mechanism by which MAGE proteins enhance the E3 ligase activities of RING proteins still remains to be uncovered.

\section{CONCLUSIONS}

Accumulating data suggested that type I MAGE proteins played an important role in the process of tumorigenesis and tumor cell viability (Duan et al., 2003; Yang et al., 2007). Importantly, MAGE proteins have also been implicated in protein degradation; however, the underlying mechanism is still unknown. For example, NECDIN has been reported to stimulate the degradation of the critical hypoxia response protein hypoxia-inducible factor $1 \alpha$ (HIF1a) through a proteasome-dependent mechanism (Moon et al., 2005). Furthermore, MAGE-D1 stimulates the ubiquitination and degradation of the anti-apoptotic factor CHE-1 (Di Certo et al., 2007). In addition to the functions of MAGE proteins in protein degradation, several MAGE-associated E3 RING ubiquitin ligases are implicated in the degradation of important cellular targets. For example, LNX1, the E3 RING ubiquitin ligase that binds MAGE-B18, promotes the ubiquitination and proteasome degradation of the p53 inhibitor NUMB (Nie et al., 2002; Colaluca et al., 2008). In addition, MAGE-D1 associated IAP proteins have multifaceted roles in the regulation of apoptosis, several of which involve the ubiquitination of substrates (Varfolomeev and Vucic, 2008). It will be interesting to examine whether the binding MAGE proteins also stimulate the ubiquitin ligase activity of these RING domain E3s, as well as many other uncharacterized RING domain E3 proteins that bind MAGEs.

MAGE cancer testis antigens have been targeted by antibody and vaccine therapies due to their specific antigen presentation on cancer cells (Brichard and Lejeune, 2007; Goldman and DeFrancesco, 2009). However, due to the lack of knowledge of how MAGE cancer testis antigens function in cells, the development of inhibitors of MAGE cancer testis antigens within cells has been unexplored. The subject matter we review here is attractive in that MAGE cancer antigens might facilitate the survival of cancer cells by binding, thus promoting TRIM28-mediated ubiquitination and proteasomal degradation of the critical tumor suppressor p53 (Doyle et al., 2010).

However, the mechanism by which the E3 ubiquitin ligase activity of TRIM28 is specifically activated by MAGEs in tumor cells is not fully understood. The hint that MAGE-C2 binds to E2 conjugating enzyme appears to contribute to two attractive 
models. However, is the binding of MAGE proteins to E2 molecules conserved among the MAGE family, or do different MAGE proteins utilize the same mechanism to enhance E3 ubiquitin ligase activities of their RING partners? Exciting answers await us when structural and biochemical studies into other MAGE-RING complexes take their shapes.

Another key question is whether the association of type I MAGEs with RING domain proteins and the enhancement of E3 ubiquitin ligase activity do confer tumor cell survival (Espantman and O'Shea, 2010). To solve this issue, the similarities and differences between germline cells and cancer cells must be investigated and the aberrant situation in tumor cells (Simpson et al., 2005), such as the expression levels of several RING E3 ligases, might be different from that of normal cells. Of interest, some TRIM family proteins were identified to be involved in MAGE-RING complexes (Rual et al., 2005; Doyle et al., 2010). The TRIM family constitutes a large E3 ubiquitin ligase family involved in a variety of cellular processes, including apoptosis, cell proliferation, cell cycle regulation and viral response (Meroni and Diez-Roux, 2005). It remains to be investigated whether other TRIM family proteins interact with MAGEs. Future structural and biochemical studies to address these questions will definitely bring forward our knowledge of the biological functions of MAGE proteins and at the same time cast more new light on the research into novel anti-cancer drugs targeted at MAGERING complexes.

\section{ACKNOWLEDGEMENTS}

This work was supported by NSFC 31030020 to Maojun Yang.

\section{ABBREVIATIONS}

co-IP, co-immunoprecipitation; HIF1 $\alpha$, hypoxia-inducible factor $1 \alpha$; IAP, inhibitor of apoptosis; MHD, MAGE homology domain; TAP, tandem affinity purification

\section{REFERENCES}

Barker, P.A., and Salehi, A. (2002). The MAGE proteins: emerging roles in cell cycle progression, apoptosis, and neurogenetic disease. J Neurosci Res 67, 705-712.

Bolli, M., Kocher, T., Adamina, M., Guller, U., Dalquen, P., Haas, P., Mirlacher, M., Gambazzi, F., Harder, F., Heberer, M., et al. (2002). Tissue microarray evaluation of Melanoma antigen E (MAGE) tumor-associated antigen expression: potential indications for specific immunotherapy and prognostic relevance in squamous cell lung carcinoma. Ann Surg 236, 785-793, discussion 793.

Borden, K.L. (2000). RING domains: master builders of molecular scaffolds? J Mol Biol 295, 1103-1112.

Brasseur, F., Rimoldi, D., Liénard, D., Lethé, B., Carrel, S., Arienti, F., Suter, L., Vanwijck, R., Bourlond, A., Humblet, Y., et al. (1995). Expression of MAGE genes in primary and metastatic cutaneous melanoma. Int J Cancer 63, 375-380.

Brichard, V.G., and Lejeune, D. (2007). GSK's antigen-specific cancer immunotherapy programme: pilot results leading to
Phase III clinical development. Vaccine 25, B61-B71.

Chomez, P., De Backer, O., Bertrand, M., De Plaen, E., Boon, T., and Lucas, S. (2001). An overview of the MAGE gene family with the identification of all human members of the family. Cancer Res 61, 5544-5551.

Cilensek, Z.M., Yehiely, F., Kular, R.K., and Deiss, L.P. (2002). A member of the GAGE family of tumor antigens is an anti-apoptotic gene that confers resistance to Fas/CD95/APO-1, Interferongamma, taxol and gamma-irradiation. Cancer Biol Ther 1, 380-387.

Colaluca, I.N., Tosoni, D., Nuciforo, P., Senic-Matuglia, F., Galimberti, V., Viale, G., Pece, S., and Di Fiore, P.P. (2008). NUMB controls p53 tumour suppressor activity. Nature 451, 76-80.

De Smet, C., Courtois, S.J., Faraoni, I., Lurquin, C., Szikora, J.P., De Backer, O., and Boon, T. (1995). Involvement of two Ets binding sites in the transcriptional activation of the MAGE1 gene. Immunogenetics 42, 282-290.

Di Certo, M.G., Corbi, N., Bruno, T., lezzi, S., De Nicola, F., Desantis, A., Ciotti, M.T., Mattei, E., Floridi, A., Fanciulli, M., et al. (2007). NRAGE associates with the anti-apoptotic factor Che-1 and regulates its degradation to induce cell death. J Cell Sci 120, 1852-1858.

Doyle, J.M., Gao, J., Wang, J., Yang, M., and Potts, P.R. (2010). MAGE-RING protein complexes comprise a family of E3 ubiquitin ligases. Mol Cell 39, 963-974.

Duan, Z., Duan, Y., Lamendola, D.E., Yusuf, R.Z., Naeem, R., Penson, R.T., and Seiden, M.V. (2003). Overexpression of MAGE/ GAGE genes in paclitaxel/doxorubicin-resistant human cancer cell lines. Clin Cancer Res 9, 2778-2785.

Espantman, K.C., and O'Shea, C.C. (2010). aMAGEing new players enter the RING to promote ubiquitylation. Mol Cell 39, 835-837.

Goldman, B., and DeFrancesco, L. (2009). The cancer vaccine roller coaster. Nat Biotechnol 27, 129-139.

Jackson, P.K., Eldridge, A.G., Freed, E., Furstenthal, L., Hsu, J.Y., Kaiser, B.K., and Reimann, J.D. (2000). The lore of the RINGs: substrate recognition and catalysis by ubiquitin ligases. Trends Cell Biol 10, 429-439.

Jordan, B.W., Dinev, D., LeMellay, V., Troppmair, J., Gotz, R., Wixler, L., Sendtner, M., Ludwig, S., and Rapp, U.R. (2001). Neurotrophin receptor-interacting mage homologue is an inducible inhibitor of apoptosis protein-interacting protein that augments cell death. $J$ Biol Chem 276, 39985-39989.

Kendall, S.E., Battelli, C., Irwin, S., Mitchell, J.G., Glackin, C.A., and Verdi, J.M. (2005). NRAGE mediates p38 activation and neural progenitor apoptosis via the bone morphogenetic protein signaling cascade. Mol Cell Biol 25, 7711-7724.

Liu, Y., Zhu, Q., and Zhu, N. (2008). Recent duplication and positive selection of the GAGE gene family. Genetica 133, 31-35.

López-Sánchez, N., González-Fernández, Z., Niinobe, M., Yoshikawa, K., and Frade, J.M. (2007). Single mage gene in the chicken genome encodes CMage, a protein with functional similarities to mammalian type II Mage proteins. Physiol Genomics 30, 156-171.

Lorick, K.L., Jensen, J.P., Fang, S., Ong, A.M., Hatakeyama, S., and Weissman, A.M. (1999). RING fingers mediate ubiquitinconjugating enzyme (E2)-dependent ubiquitination. Proc Natl Acad Sci U S A 96, 11364-11369.

Meroni, G., and Diez-Roux, G. (2005). TRIM/RBCC, a novel class of 'single protein RING finger' E3 ubiquitin ligases.Bioessays 27, 
1147-1157.

Miranda, E.I. (2010). MAGE, biological functions and potential clinical applications. Leuk Res 34, 1121-1122.

Moon, H.E., Ahn, M.Y., Park, J.A., Min, K.J., Kwon, Y.W., and Kim, K. W. (2005). Negative regulation of hypoxia inducible factor-lalpha by necdin. FEBS Lett 579, 3797-3801.

Nasmyth, K., and Haering, C.H. (2005). The structure and function of SMC and kleisin complexes. Annu Rev Biochem 74, 595-648.

Nie, J., McGill, M.A., Dermer, M., Dho, S.E., Wolting, C.D., and McGlade, C.J. (2002). LNX functions as a RING type E3 ubiquitin ligase that targets the cell fate determinant Numb for ubiquitindependent degradation. EMBO J 21, 93-102.

Ohman Forslund, K., and Nordqvist, K. (2001). The melanoma antigen genes-any clues to their functions in normal tissues? Exp Cell Res 265, 185-194.

Park, J.H., Kong, G.H., and Lee, S.W. (2002). hMAGE-A1 overexpression reduces TNF-alpha cytotoxicity in ME-180 cells. Mol Cells 14, 122-129.

Patard, J.J., Brasseur, F., Gil-Diez, S., Radvanyi, F., Marchand, M., François, P., Abi-Aad, A., Van Cangh, P., Abbou, C.C., Chopin, D., et al. (1995). Expression of MAGE genes in transitional-cell carcinomas of the urinary bladder. Int $\mathrm{J}$ Cancer 64, 60-64.

Pawson, T., and Nash, P. (2003). Assembly of cell regulatory systems through protein interaction domains. Science 300, 445-452.

Pebernard, S., McDonald, W.H., Pavlova, Y., Yates, J.R. 3rd, and Boddy, M.N. (2004). Nse1, Nse2, and a novel subunit of the Smc5Smc6 complex, Nse3, play a crucial role in meiosis. Mol Biol Cell 15, 4866-4876.

Pebernard, S., Perry, J.J., Tainer, J.A., and Boddy, M.N. (2008). Nse1 RING-like domain supports functions of the Smc5-Smc6 holocomplex in genome stability. Mol Biol Cell 19, 4099-4109.

Potts, P.R. (2009). The Yin and Yang of the MMS21-SMC5/6 SUMO ligase complex in homologous recombination. DNA Repair (Amst) 8, 499-506.

Rual, J.F., Venkatesan, K., Hao, T., Hirozane-Kishikawa, T., Dricot, A., Li, N., Berriz, G.F., Gibbons, F.D., Dreze, M., Ayivi-Guedehoussou, N., et al. (2005). Towards a proteome-scale map of the human protein-protein interaction network. Nature 437, 1173-1178.

Sasaki, A., Masuda, Y., Iwai, K., Ikeda, K., and Watanabe, K. (2002). A RING finger protein Praja1 regulates DIx5-dependent transcription through its ubiquitin ligase activity for the Dlx/Msxinteracting MAGE/Necdin family protein, Dlxin-1. J Biol Chem 277, 22541-22546.

Scanlan, M.J., Gure, A.O., Jungbluth, A.A., Old, L.J., and Chen, Y.T. (2002). Cancer/testis antigens: an expanding family of targets for cancer immunotherapy. Immunol Rev 188, 22-32.

Scanlan, M.J., Simpson, A.J., and Old, L.J. (2004). The cancer/testis genes: review, standardization, and commentary. Cancer Immun $4,1$.

Sergeant, J., Taylor, E., Palecek, J., Fousteri, M., Andrews, E.A., Sweeney, S., Shinagawa, H., Watts, F.Z., and Lehmann, A.R. (2005). Composition and architecture of the Schizosaccharomyces pombe Rad18 (Smc5-6) complex. Mol Cell Biol 25, 172-184.

Simpson, A.J., Caballero, O.L., Jungbluth, A., Chen, Y.T., and Old, L. J. (2005). Cancer/testis antigens, gametogenesis and cancer. Nat Rev Cancer 5, 615-625.

Taylor, E.M., Copsey, A.C., Hudson, J.J., Vidot, S., and Lehmann, A. R. (2008). Identification of the proteins, including MAGEG1, that make up the human SMC5-6 protein complex. Mol Cell Biol 28, 1197-1206.

van der Bruggen, P., Traversari, C., Chomez, P., Lurquin, C., De Plaen, E., Van den Eynde, B., Knuth, A., and Boon, T. (1991). A gene encoding an antigen recognized by cytolytic $T$ lymphocytes on a human melanoma. Science 254, 1643-1647.

Varfolomeev, E., and Vucic, D. (2008). (Un)expected roles of c-IAPs in apoptotic and NFkappaB signaling pathways. Cell Cycle 7, 1511-1521.

Wang, C., Ivanov, A., Chen, L., Fredericks, W.J., Seto, E., Rauscher, F.J. 3rd, and Chen, J. (2005). MDM2 interaction with nuclear corepressor KAP1 contributes to p53 inactivation. EMBO J 24, 3279-3290.

Yang, B., O'Herrin, S.M., Wu, J., Reagan-Shaw, S., Ma, Y., Bhat, K. M., Gravekamp, C., Setaluri, V., Peters, N., Hoffmann, F.M., et al. (2007). MAGE-A, mMage-b, and MAGE-C proteins form complexes with KAP1 and suppress p53-dependent apoptosis in MAGE-positive cell lines. Cancer Res 67, 9954-9962.

Zheng, N., Schulman, B.A., Song, L., Miller, J.J., Jeffrey, P.D., Wang, P., Chu, C., Koepp, D.M., Elledge, S.J., Pagano, M., et al. (2002). Structure of the Cul1-Rbx1-Skp1-F boxSkp2 SCF ubiquitin ligase complex. Nature 416, 703-709. 